\title{
Intérêt des multicouches constituées de matériaux légers pour la réalisation d'optiques X-UV autour de $40 \mathrm{eV}$
}

\author{
F. Bridou, M.F. Ravet, F. Delmotte, X. Song et A. Jerome \\ Laboratoire Charles Fabry de l'Institut d'Optique, UMR 8501 du CNRS, bâtiment 503, \\ Centre Scientifique d'Orsay, 91403 Orsay cedex, France
}

\begin{abstract}
Résumé : Dans la gamme de longueur d'onde des X-UV (3-40 nm ou $400-30 \mathrm{eV})$, il est possible de réaliser des optiques à partir de miroirs revêtus de multicouches constituées de matériaux alternés, dont l'un est plus spécifiquement réfléchissant (habituellement de numéro atomique élevé), et l'autre plus spécifiquement "espaceur " (de numéro atomique faible), de manière à ce que dans la période élémentaire constituée par la bicouche, les interférences soient en phase pour la longueur d'onde et l'angle d'incidence considéré. Ces deux matériaux sont habituellement désignés par "lourd" et "léger". On montre quelques exemples où, selon la longueur d'onde d'utilisation, le matériau dit "lourd" peut être remplacé avantageusement par un élément de faible numéro atomique.
\end{abstract}

\section{INTRODUCTION}

L'association de miroirs sphériques (ou asphériques) revêtus de multicouches adaptées permet la réalisation de systèmes catadioptriques imageurs autour d'une longueur d'onde bien définie, par exemple : télescopes spatiaux (images du soleil), microscopes (images de plasmas lasers).

Dans la gamme X-UV située entre 30 et $400 \mathrm{eV}$, pour obtenir la meilleure réflectivité possible dans un empilement multicouche, on sélectionne deux matériaux ayant un contraste d'indice élevé (correspondant au maximum d'écart entre les parties réelles), et dont les absorptions (correspondant aux parties imaginaires) sont les plus faibles possibles (figure 1).

Pour $\bullet>130 \AA$, deux matériaux de prédilection : Mo pour le matériau "lourd" et Si pour le matériau "léger" sont utilisés depuis de nombreuses années, tant pour leur efficacité, que pour leur stabilité.

Selon le domaine d'énergie plus particulièrement concerné, et en fonction de leurs indices, d'autres couples de matériaux peuvent être envisagés, et en particulier des éléments à faible numéro atomique.
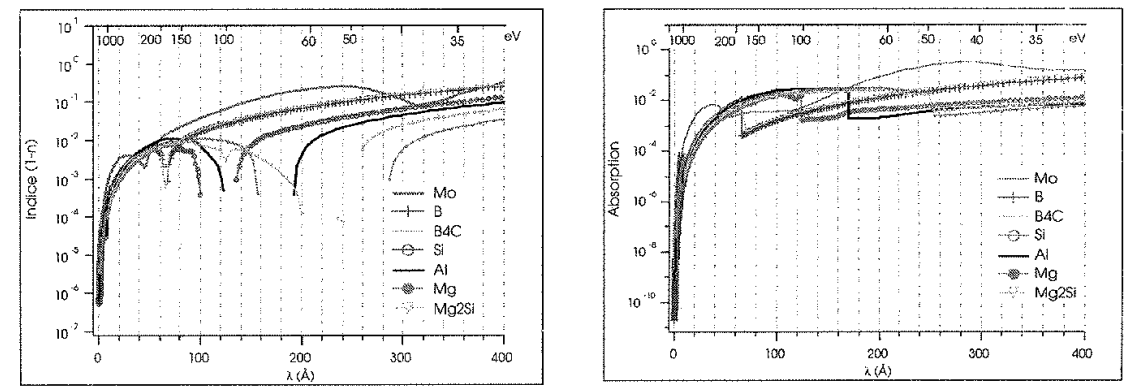

Figure 1: Indices complexes de quelques matériaux (parties réelles et imaginaires):

"Légers": SILICIUM, ALUMINIUM, MAGNESIUM,SILICIURE DE MAGNESIUM,

"Lourds": MOLYBDENE, BORE, CARBURE DE BORE, Calculés à partir des tables de HENKE [ref I].

\section{EXEMPLES D'EMPILEMENTS DE MATERIAUX “légers" (Calculs)}

En général on cherche à maximiser la réflectivité de l'empilement en augmentant le nombre de périodes, (ce que permet une absorption relativement faible pour les deux matériaux), et en optimisant le rapport des épaisseurs des deux composants.

D'autres applications nécessitent la réalisation de filtres spectraux. Dans ce cas on souhaite optimiser la sélectivité en longueur d'onde de la multicouche. Ceci peut être réalisé en diminuant l'épaisseur du matériau absorbant, ce qui permet d'augmenter le nombre de couches participant efficacenent à la réflexion et de réduire la bande passante $\Delta \lambda$.

Pour les trois longueurs d'ondes considérées 171 (raie d'émission FeIX/X), 304 (raie He II) et $401 \AA$ (raie $\mathrm{Ni}$ $\mathrm{VI}$ ), on compare les calculs de réflectivité que l'or obtient pour $\mathrm{Mo} / \mathrm{Si}$, avec ceux que l'on obticnt avec des matériaux légers. On voit que ces demiers peuvent avoir de meilleures performances (figures 2 ๖). 
Les figures de gauche correspondent à une optimisation de la réflectivité, celles de droite, à une optimisation de la sélectivité.

\section{1 Longueur d'onde d'utilisation: $\lambda=171 \AA$}

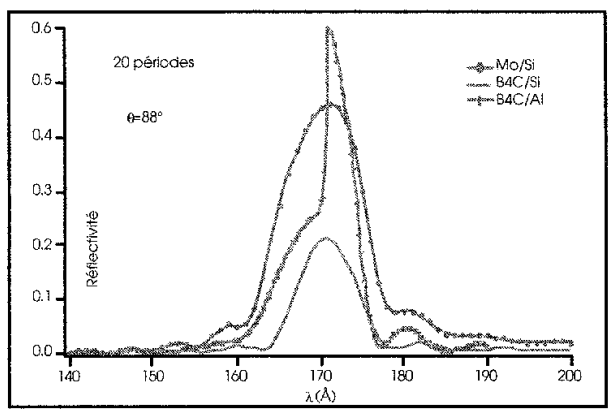

(a)

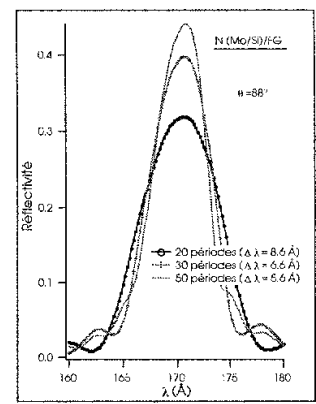

(b)

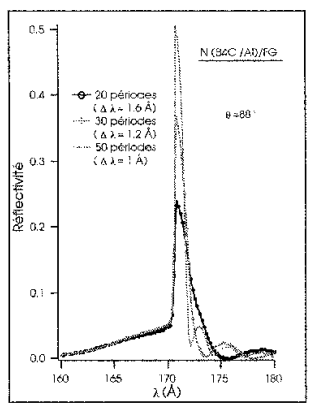

(c)

Figure 2 : a) Calcul de la réflectivité pour 20 périodes de multicouches $\mathrm{Mo} / \mathrm{Si}, \mathrm{B}_{4} \mathrm{C} / \mathrm{Si}$ et $\mathrm{B}{ }_{4} \mathrm{C} / \mathrm{Al}$, dont le rapport $\gamma$ de l épaisseur du matériau réfléchissant sur l'épaisseur de la période a été optimisé. La rugosité rms est de $6 \AA$ pour toutes les interfaces. b) Sélectivité (largeur à mi-hauteur $\Delta \lambda$ ) obtenue pour un nombre de périodes $\mathrm{Mo} / \mathrm{Si}$ crossant . c) Sélectivité obtenue pour un nombre de périodes $\mathrm{B}_{4} \mathrm{C} / \mathrm{Al}$ croissant.

\subsection{Longueur d'onde d'utilisation: $\lambda=304 \AA$}
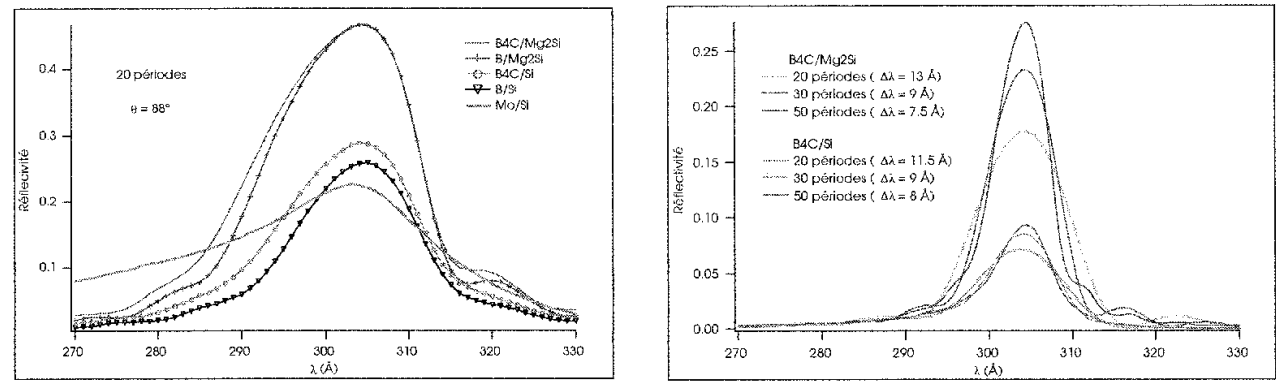

Figure $3:$ a) Comparaison des réflectivités calculées pour 20 périodes de multicouches $\mathrm{Mo} / \mathrm{Si}, \mathrm{B} / \mathrm{Si}, \mathrm{B}_{4} \mathrm{C} / \mathrm{Si}$, $\mathrm{B} / \mathrm{Mg} 2 \mathrm{Si}$ et $\mathrm{B}_{4} \mathrm{C} / \mathrm{Mg}_{2} \mathrm{Si}$ dont le $\gamma$ a été optimisé. (Rugosité $\mathrm{rms}=6 \AA$ pour toutes les interfaces).

b) Comparaison des sélectivités calculées pour $\mathrm{B}_{4} \mathrm{C} / \mathrm{Si}$ et $\mathrm{Mg}_{2} \mathrm{Si} / \mathrm{B}_{4} \mathrm{C}$ en réduisant $\gamma$.

\subsection{Longueur d'onde d'utilisation: $\lambda=401 \AA$}
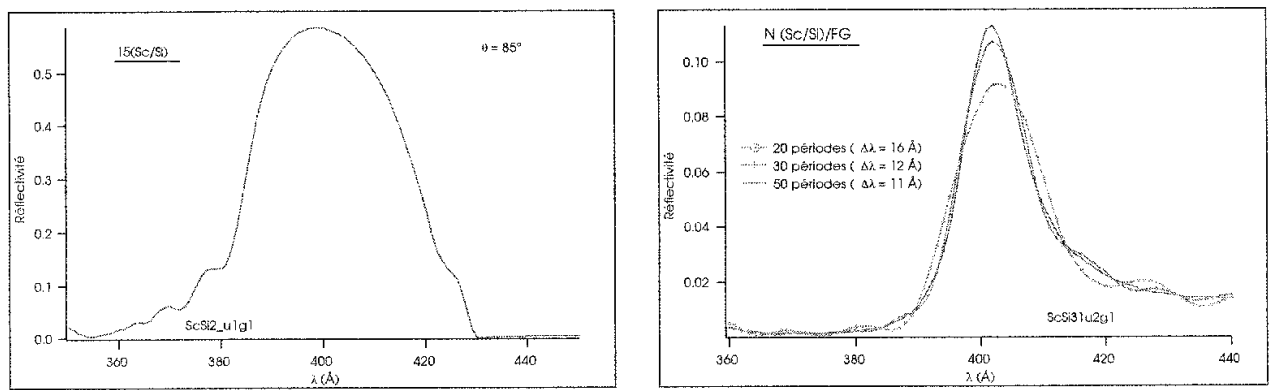

Figure 4: a) Réflectivité d'une multicouche $\mathrm{Sc} / \mathrm{Si}$ de 15 périodes calculée à partir des indices de Windt [2].

b) La sélectivité obtenue en diminuant le rapport $\gamma$ reste limitée du fait que l'absorption des deux matériaux devient non négligeable pour cette longueur d'onde. 


\section{EXEMPLES DE REALISATIONS}

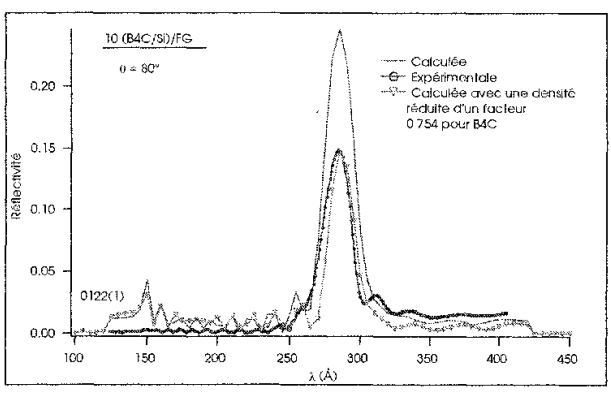

Figure 5 :Multicouche $\mathrm{B}_{4} \mathrm{C} / \mathrm{Si}$ autour de $284 \AA$

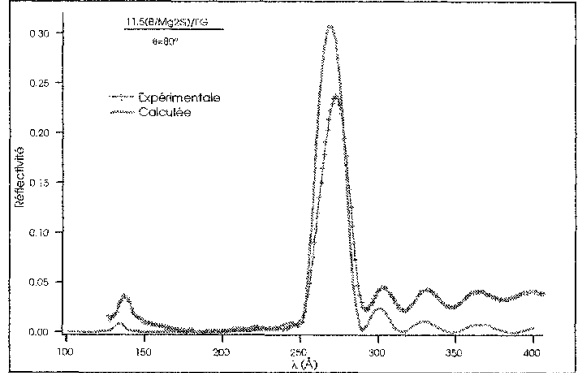

Figure 6 : Multicouche B/Mg2Si autour de $284 \AA$

La réalisation de ces multicouches a fait apparaître plusieurs problèmes:

- Instabilité, matériaux sensibles à l'oxydation : $\mathrm{B}, \mathrm{Al}, \mathrm{Mg}$.

- Décollement des multicouches (adhérence, contraintes): $\mathrm{B}_{4} \mathrm{C}$.

- Indices effectifs des matériaux déposés mal connus, différents du massif, notamment $\mathrm{B}_{4} \mathrm{C}$.

Des améliorations sont possibles:

- Etude des couches de protection en surface ou dans l'empilement (développée en [3]).

- Réduction des contraintes par optimisation des épaisseurs et/ou des conditions de dépôt.

- Etude approfondie de la composition des matériaux déposés .

\section{CONCLUSION}

Les matériaux légers dans les multicouches réfléchissantes offrent de nouvelles perspectives dans le cadre de la réalisation d 'optiques pour rayons $X$ dans la gamme de longueur d 'onde comprise entre 150 et $500 \AA$ ( 25 et 85 eV). La réalisation de ces empilements nécessite des études spécifiques notamment pour en améliorer la stabilité.

\section{REFERENCES}

[1] B.L. Henke, E. _M. Gullikson, J. C. Davis, At. Data Nucl. Data Tables 54, 181 (1993) et http://wwwcxro.lbl.gov/optical_constants/

[2] http://cletus.phys.columbia.edu/ windt/

[3] Dépôt et calibration de multicouches pour l 'optique X-UV dans la gamme 10-30 nm, F.Delmotte et col,, cette conférence

[4] Réflectivité et sélectivité spectrale de multicouches B/Si dans le domaine des X-UV. Approche théorique et expérimentale, F.Bridou, M.-F. Ravet, A. Raynal, J.-P. Chauvineau, B. Agius, Ph. Troussel, J. Phys IV France 9 (1999), Pr5-77.

[4] B/Si multilayers for soft X-ray and extreme ultraviolet optics, M.-F. Ravet, F. Bridou, A. Raynal, B. Pardo, J.-P. Chauvineau, J.-M. André, J. Appl. Phys. 89, 1145 (2001).

[5] Multilayers based on light materials for optics in the $30 \mathrm{~nm}$ wavelength region, M.F. Ravet, F, Bridou, F. Delmotte, A. Jerome, J.-P. Delaboudinière, X. Song, M. Bougnet,

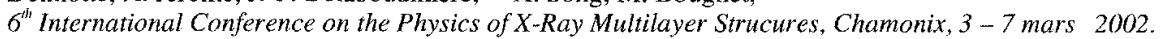

